\title{
Improved silver halide crystals for photographic emulsion
}

\author{
T. I. Abdullin ${ }^{1}$, V. P. Andrianov ${ }^{2}$, Yu. V. Badeev ${ }^{1}$, Yu. A. Breslav ${ }^{2}$, E. M. Giljazetdinov', \\ A. N. Golubev ${ }^{1}$, B. G. Hayatov ${ }^{2}$, S. A. Koshkin ${ }^{1}$, I. E. Mumdji ${ }^{1}$, S. I. Nikitin ${ }^{1}$, Yu. G. Shtyrlin ${ }^{1}$, \\ Yu. A. Zakharov"
}

${ }^{1}$ Kazan (Volga region) Federal University, 18 Kremlyovskaya st. Kazan, Russia

${ }^{2}$ Scientific and Production Enterprise "Tasma", Vosstaniya str., 100, Kazan, Russia

\author{
Email address: \\ yuri.zakharov@ksu.ru (Yu. A. Zakharov)
}

\section{To cite this article:}

T. I. Abdullin, V. P. Andrianov, Yu. V. Badeev, Yu. A. Breslav, E. M. Giljazetdinov, A. N. Golubev, B. G. Hayatov, S. A. Koshkin, I. E. Mumdji, S. I. Nikitin, Yu. G. Shtyrlin, Yu. A. Zakharov. Improved Silver Halide Crystals for Photographic Emulsion, Advances in Materials. Vol. 1, No. 1, 2012, pp. 16-19. doi: 10.11648/j.am.20120101.13

\begin{abstract}
The computer controlled multistage synthesis of fine-grained photographic emulsions is described. Scanning electron microscopy was used to investigate the shape and size $(10-1500 \mathrm{~nm})$ distribution of silver halide crystals in the emulsion. The influence of stirrers in the chemical reactor and also surfactant addition on the emulsion quality was revealed. In the result of the investigation a new method for obtaining of high quality photosensitive materials based on the tabular multicoated silver halide crystals was proposed.
\end{abstract}

Keywords: Photographic Emulsion, Silver Halide Microcrystals, Surfactant

\section{Introduction}

Emulsions based on highly homogeneous tabular T-shaped crystals or flat microcrystals (MC) of the silver halide AgHal are raw material for production of modern photographic films [1], in particular, for X-ray technical and medical photographic applications. The influence of size and shape of the MC on sensitivity, resolution, contrast and other parameters of the films is very strong. Granulometric homogeneity (CV) is usually used for characterization of the synthesized emulsion quality before the final stage of a photographic films production. $\mathrm{CV}$ is a ratio of the standard deviation of the $\mathrm{MC}$ equivalent diameter to its arithmetic mean. This statistical analysis should be performed for at least 300 particles. On the other hand, CV and crystals shape significantly depend on several factors: design of the chemical reactor, a gelatin solution mixer, a method for introducing the reagent solutions, a type of gelatin, etc. Modern synthesis of AgHal MC has 20 variables that must withstand at well-defined algorithms [2]. It is a complicated technological problem for an industrial production. Therefore, obtaining the desired properties of emulsions and providing their reproducibility is the actual problem up to now.

Several years ago the effectiveness of high homogeneous $(\mathrm{CV} \leqslant 20 \%)$ and extrahomogeneous $(\mathrm{CV} \leqslant 10 \%)$ $\mathrm{T}$-crystals in X-ray technical photographic films was shown by R. Dickerson and A. Tsor $[3,4]$. The silver consumption in the film production was reduced up to $25 \%$ without loss of the photographic sensitivity compared with emulsions made of thickened or isometric MC. Surface-active agents (surfactants) under the trade mark Pluronic two-functional polyols: polyoxy-propylene polyethylene glycols (PPG-PEG-PPG, Mn 3300), polyoxyethylene poly-propylene glycols (PEG-PPG-PEG, Mn 2000); and Tetronic - alkoxylated tetrafunctional ethylenediamine were proposed [3,5-7] to improve the T-crystals uniformity. The optimal concentration of the surfactant was $1-5 \%$ from silver nitrate introduced into the reactor at the emulsion nucleation stage. Unfortunately, this high surfactant concentrations in the process of industrial film production increase the veil. Now new brands of surfactants entered the market of chemicals. Photographic films manufacturers have practical interest in using of more effective surfactants for obtaining high homogeneous low-veil emulsions.

The aim of this work is description of the synthesis procedure for multicoated tabular AgHal crystals obtained utilizing different mixing devices and new surfactant (three-functional copolymer of propylene oxide and ethylene oxide based on glycerol, the brand Laprol 3603-2-12, TU 2226-015-10488057-94 of JSC "Nizhnekamskneftekhim", Russia).

\section{Materials and Methods}


Synthesis of the photographic emulsions was carried out in the laboratory homemade setup for two-jet crystallization. The setup consists of the 2 liters capacity chemical reactor equipped with computer-controlled thermostat system and peristaltic pumps (for supplying of reagents into the gelatin solution). We used two types of mixing devices - simple propeller stirrer and special homemade stirrer [8]. Last model was settled down in the lower part of the reactor and consists of a turbine and a propeller inside the tube case with conic bells. Liquid reagents were pumped from narrow silicone tubes under the turbine. The stream of the gelatin solution was directed upward. Therefore our reagents were mixed up very quickly and reacted with each other inside the tube case. Afterwards they were ejected into the volume of the reactor and repeatedly circulated via the mixing device. The temperature and $\mathrm{pBr}$ of the emulsion were controlled automatically using feedback from thermal and ion sensors to the corresponding pumps. Operation of the pumps and the data from all sensors were automatically recorded [2]. Such procedure provided high reproducibility of the conditions for the emulsion synthesis. We varied only concentration of the surfactant addition $(0$; 0,$005 ; 0,01 ; 0,05 ; 0,5 \%$ wt. of Laprol per amount of AgNO3 entered into the reactor at the nucleation stage).

Synthesis of the fine-grained emulsion for high resolution photographic films was carried out according to the following scheme:

Loading of the reactor: $675 \mathrm{ml}$ of initial gelatin solution in water with concentration of the gelatin $0,8 \%$ wt.; Laprol in necessary concentration; the water solution of $\mathrm{KBr}$ up to $\mathrm{pBr}=1,3$ in the final gelatin mixture (solution was mixed carefully and heated up to $40 \mathrm{oC}$ ).

The 1st stage - nucleation: solutions of 2,0 $\mathrm{M}$ of $\mathrm{AgNO} 3$ and $2,0 \mathrm{M}$ of $\mathrm{KBr}$ with rates of $6,75 \mathrm{ml} / \mathrm{min}$ and 10,7 $\mathrm{ml} / \mathrm{min}$, respectively, were injected during $125 \mathrm{~s}$. Rotation rate of the stirrer was $600 \mathrm{rpm}$. Afterwards, the temperature of the emulsion was increased up to $50{ }^{\circ} \mathrm{C}$ during $20 \mathrm{~min}$. The $\mathrm{pBr}$ value was increased up to 1,6 by injection of 2,0 $\mathrm{M}$ of AgNO3 solution with rate of $0,85 \mathrm{ml} / \mathrm{min}$. Then, $\mathrm{pH}$ of the emulsion was tuned up to 9,6 by addition of $25 \% \mathrm{wt}$. of the ammonia water solution.

The 2nd stage - Ostwald ripening: this stage was carried out at $50{ }^{\circ} \mathrm{C}$ within $12 \mathrm{~min}$. Afterwards the emulsion was acidified to $\mathrm{pH} 5,5$ by acetic acid injection. $100 \mathrm{ml}$ of $13 \%$ wt. of gelatin solution in water were added into the reactor.

The 3rd stage - crystallization of the 1st coat: solutions of 2,0 M AgNO3 and 2,0 M KBr + 0,03 M KI were injected with equal rates $1,489+0,17 \mathrm{t} \mathrm{ml} / \mathrm{min}$ during $\mathrm{t}=32 \mathrm{~min}$ at $\mathrm{pBr}=1,6$. The rotation rate of the stirrer was $800 \mathrm{rpm}$.

The 4th stage - crystallization of the 2nd coat: solutions of 2,0 $\mathrm{M} \mathrm{AgNO} 3$ and 2,0 $\mathrm{M}$ of $\mathrm{KBr}$ were injected with rates $6,008+0,13 \mathrm{t} \mathrm{ml} / \mathrm{min}$ during $\mathrm{t}=30 \mathrm{~min}$ at $\mathrm{pBr}=2,0$. The rotation rate of the stirrer was $1000 \mathrm{rpm}$.

After the end of the synthesis MC were separated from gelatin by centrifugation and washing in the distilled water with ultrasound processing. About $5 \mu \mathrm{l}$ of final water suspension of microcrystals were dried on a fresh plate of mica for the scanning electronic microscope (SEM) measurements. We used Merlin SEM (Carl Zeiss). Set of SEM images with magnification X10 000 for MC at the mica plate surface was obtained. Magnification X20 000 was used for analyzing the crystal shape.

Critical concentration of the micelle formation (CCM) by Laprol was determined by the method [9] using the dependence of pyrene fluorescence intensity at $373 \mathrm{~nm}$ on the Laprol concentration. For the fluorescence measurements Microplate reader Infinite 200 PRO (Tecan) was used (excitation wavelength was equal to $339 \mathrm{~nm}$ ).

\section{Results and Discussion}

We have chosen the above synthesis route because it provides obtaining the fine-grained homogeneous emulsion of tabular $\mathrm{MC}$ with the required average equivalent diameter of about $0.5 \mu \mathrm{m}$ and $\mathrm{C}_{\mathrm{V}} \leq 30 \%$ if a special mixing device is used [8]. The SEM image of such MC population is shown in Fig. 1. Actually, the experiment with simple propeller stirrer has firstly been done. It shows a role of mixing device in obtaining the high quality emulsions.

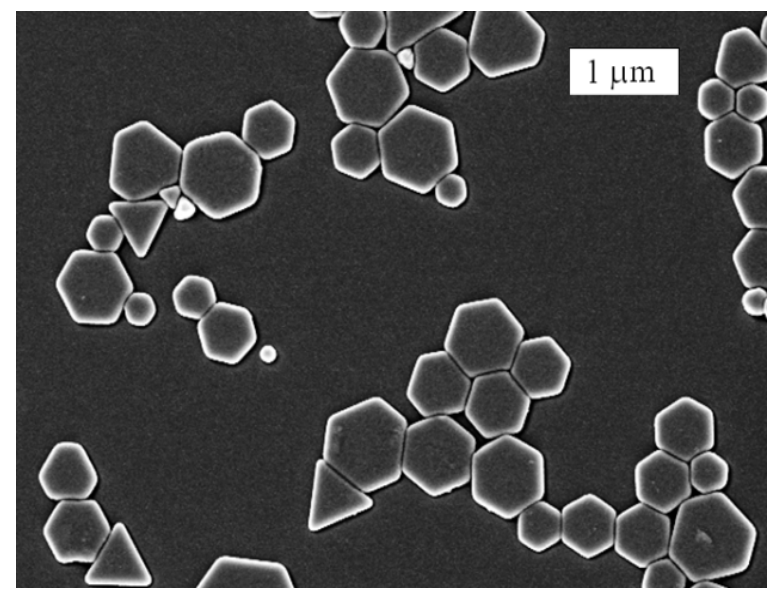

Figure 1. The SEM image of MC population if special mixing device [8] is used for the emulsion synthesis.

Fig. 2a shows MC of the emulsion which was obtained using the simple propeller stirrer without surfactant. It is not quite homogeneous. Corresponding histogram shows that the population consists of a small fraction of relatively large T-crystals with equivalent diameter $1.3 \mu \mathrm{m}$ and a large number of small, about $0.2 \mu \mathrm{m}$ crystals of spherical shape. The thickness of the large MCs varies from 0.15 to $0.28 \mu \mathrm{m}$. The granulometric homogeneity of such mixture of particles is very low $\mathrm{CV}=84 \%$. It is not suitable for high-quality photographic films. It is interesting to see the action of Laprol as a potential amplifier of the T-crystals homogeneity in the conditions for obtaining the low-grade emulsion. Successful result will be especially important for removing of any destabilizing factors which can appear during industrial synthesis. 

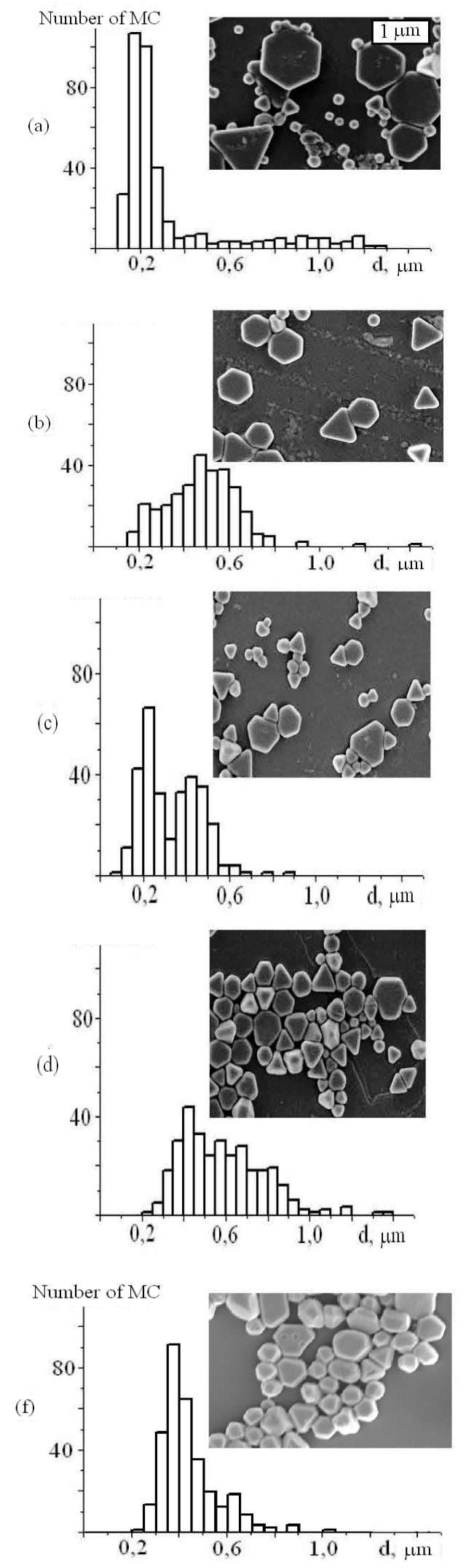

Figure 2. The SEM images and size distributions (equivalent diameter) of microcrystals AgHal synthesized at different Laprol concentrations: $a-0$; $b-0.005 ; c-0.01 ; d-0.05 ; f-0,5 \%$ wt. of $\mathrm{AgNO}_{3}$ which was used for the stage of nucleation. Magnification of all images is the same.
Theoretically, the effect of surfactants on the crystallization process is explained by formation of micelles around the MC nucleus. This shell smoothes fluctuations in the rate of increase the mass of single crystals due to the inevitable concentration and temperature gradients in the area of reagents injection and in the reactor volume as a whole.

According to our measurements, CCM of Laprol in deionized water at the temperature $40{ }^{\circ} \mathrm{C}$ is $0.042 \%$ wt. However, content of $0.8 \%$ wt. gelatin in water solution reduces this value down to $0.007 \%$ wt. Reduction of CCM can be explained by participation of the protein molecules in the formation of the colloidal system - composite micelles. Taking into account that $4.8 \mathrm{~g}$ of AgNO3 is introduced into the reactor at the stage of nucleation, and volume of gelatin solution reaches about $700 \mathrm{ml}$ at the end of this stage, we can recalculate concentrations of the surfactant additions in our experiments on the weight of final gelatin solution. It will be approximately $3,4 \times 10-5 ; 6,8 \times$ $10-5 ; 34 \times 10-5 ; 340 \times 10-5 \%$ wt., that is more than twice lower than CCM. However, the minimal surfactant addition changed the original emulsion significantly (Fig. 2b). The number of small and large particles considerably reduced. T-crystals are mainly formed with an average equivalent diameter $0,47 \mu \mathrm{m}$ and a thickness of $0,16 \pm 0,02 \mu \mathrm{m}$. Homogeneity was $\mathrm{CV}=32 \%$. There are about $70 \%$ hexagonal T-crystals and $\sim 25 \%$ of T-triangular crystals in the particle population. This emulsion is similar to the emulsion in Fig. 1 and hence, acceptable for production of high quality photographic films.

With twice increasing the Laprol addition (Fig. 2c) it is observed formation of two groups of particles in roughly equal proportions: 1 - flat crystals with an equivalent diameter 0,35-0,55 $\mu \mathrm{m}$ and thickness $0.1-0.18 \mu \mathrm{m}$, and $2-$ isometric crystals having the size $0.1-0.3 \mu \mathrm{m}$. It is so-called bimodal emulsion. The symmetry of the flat crystals is significantly disturbed. Irregular hexagons and triangles are observed. A trend of their transformation into isometric MC is observed. Homogeneity is deteriorated and equal $\mathrm{CV}=$ $40 \%$. Consequently, the given concentration of Laprol already is not optimal for high quality photographic emulsion.

The third - largest Laprol addition causes a tendency to thicken the MC and to create octahedral particles (Fig. 2d). Fine fraction of about $0.2 \mu \mathrm{m}$ almost disappeared. The number of large crystals increased again. The average size of the MC reached $0.58 \mu \mathrm{m}$. The relative share of the triangular MC increased. There is not only the deterioration of the grain size $(\mathrm{CV}=302 \%)$ but the transformation of the crystal form in this case.

Fourth Laprol concentration turns the whole emulsion MCs into octahedral shape of 0.3-0.8 $\mu \mathrm{m}$ size with sharp maximum near $0.4 \mu \mathrm{m}$ of the distribution hystogram (Fig. $2 \mathrm{f})$. In this case, the synthesis of T-crystals is impossible. The homogeneity of the emulsion $\mathrm{CV}=26 \%$, but it is not suitable for manufacturing of high-quality photographic films.

It is known hypothesis that T-crystals are formed by 
self-assembly of AgHal nuclei in flat associates and their subsequent stacked coalescence. We could confirm it having processed water suspension of microcrystals by ultrasound. Results of such intensive processing are shown in Fig. 3. Ultrasound begins to destroy the tabular crystals and to bare initial structure of conglomerated small particles.

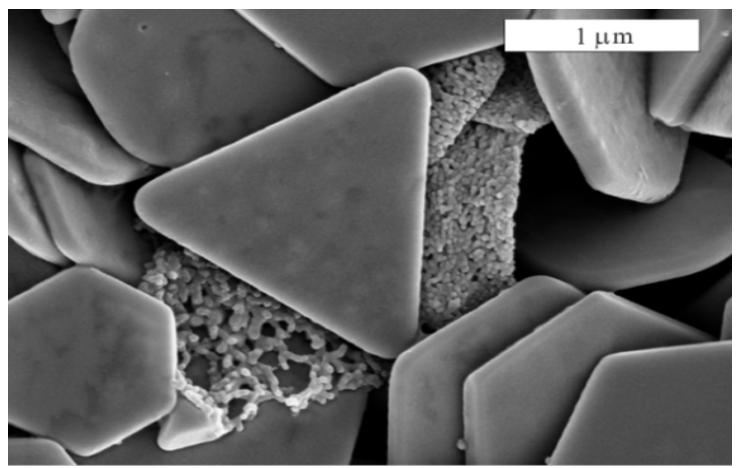

Figure 3. Revealing of the initial structure of tabular AgHal microcrystals consisting of accrete small particles.

Apparently, determined in this work micro-addition of surfactant is optimal for regulation of tabular crystals growth. Increasing of the surfactant addition by order in concentration dramatically changes the crystal morphology and shape. Instead of T-crystals the octahedral MC grow, which are not suitable for the manufacturing of high-quality photographic films.

\section{Conclusions}

Our experiments have shown that both special mixing device and surfactant Laprol additions are powerful factors to control size distribution and crystallographic structure of the silver halide microcrystals in gelatin emulsion. On the one hand, Laprol can significantly increase the homogeneity of tabular MC. This is observed when the surfactant concentration is 200 times smaller than the CCM. In terms of the initial gelatin solution it is $34 \times 10-6 \%$ wt., i.e. very small, microscopic addition to the final photographic emulsion. Therefore, it is unlikely to be a source of negative influences on the subsequent processing and properties of the photographic films. On the other hand, it was found that the properties of the final emulsion are sensitive to small (two-time) change of the micro-concentration of Laprol that significantly impairs the homogeneity. Consequently, a precise calculation of the optimal surfactant addition is required for synthesis of improved silver halide crystals for photographic emulsions.

\section{Acknowledgements}

This work was financially supported by Ministry of Education and Science of Russian Federation, Contract № 13.G25.31.0075, October 22, 2010, Resolution of the RF Government № 218.

\section{References}

[1] B.I. Shapiro, Theoretical beginnings of photographic process, Editorial URSS, Moscow 2000. 288 pages (on Russian).

[2] A.R.Garifzyanov, Yu.A.Zakharov, A.E.Klimovitsky, S.I.Nikitin, Yu.G.Shtyrlin, S.G. Gnevashev, A.N.Golubev, I.E.Mumdzhi, T.R.Sharafiyev, Yu.V.Badeev, V.P. Andrianov, E.L.Samkov, R.L. Hamzin, G. M. Sardushkina, Yu.A.Breslav, B. G. Hayatov, The automated apparatus for synthesis of photographic emulsions on the basis of silver halogenides microcrystals with significantly improved granulometric and sensitometric properties, Proceedings of International correspondence scientific and practical conference Questions of science and techniques (Novosibirsk, Russia, January 16, 2012): p. 24-31.

[3] Eastman Kodak Company, Radiographic elements with improved detective quantum efficiencies, US Patent 5252442 (1993).

[4] Eastman Kodak Company, Forehardened high aspect ratio silver halide photographic elements and processes for their use, US Patent 4414304 (1983).

[5] Agfa-Gevaert, Film/screen system and image-forming system for use in direct X-ray applications, US Patent 6528227 (2003).

[6] Eastman Kodak Company, Element for industrial radiography, US Patent 5965337 (1999).

[7] Eastman Kodak Company, Process of preparing a tabular grain emulsion having a very low coefficient of variation, European Patent 0514742 (1992).

[8] Scientific and Production Enterprise "Tasma", Kazan (Volga region) Federal University, Apparatus for the synthesis of photographic emulsions, RF Patent 2012126924 (2012).

[9] D. Wang, Z. Peng, X. Liu, Z. Tong, C. Wang, B. Ren, Synthesis and micelle formation of triblock copolymers of poly(methyl methacrylate)-b-poly(ethylene oxide)-b-poly(methyl methacrylate) in aqueous solution, Eur. Polymer J. 200743 (7): p. 2799-2808. 